\title{
Evaluation of the Patients with Urinary Tract Infection after Transrectal Ultrasound-guided Prostate Biopsy
}

\author{
Musab Ali Kutluhan, ${ }^{1}$ (1) Tuncay Toprak, ${ }^{1}$ (i) Ramazan Topaktaş ${ }^{2}$ \\ ${ }^{1}$ Department of Urology, University of Health Sciences Turkey, Fatih Sultan Mehmet Training and Research Hospital, Istanbul, Turkey \\ ${ }^{2}$ Department of Urology, University of Health Sciences Turkey, Haydarpasa Numune Training and Research Hospital, Istanbul, Turkey
}

\begin{abstract}
Introduction: This study aims to evaluate the patients who developed urinary tract infection after transrectal ultrasoundguided prostate biopsy and to determine antibiotic resistance profiles in urinary cultures after prostate biopsy.

Methods: Between 2015 and 2019, 892 patients who underwent transrectal ultrasound-guided prostate biopsy because of suspected rectal examination and/or increased PSA level were retrospectively reviewed. Urine cultures of all patients before biopsy were sterile. All patients received oral ciprofloxacin prophylaxis, starting three days before prostate biopsy. Standard 12 quadrant prostate biopsies were performed to all patients. Forty-five patients who were admitted to the hospital again due to fever, dysuria or sepsis were included in this study. In these cases, microorganisms and resistance status were investigated.

Results: Forty patients had complaints of dysuria, 17 patients had fever with tremors, and one patient had sepsis. No prostate abscess was detected on ultrasound. The rate of urinary tract infection after ciprofloxacin prophylaxis was 5.04\% and 1.90\% of the patients had fever after prostate biopsy. Reproduction was detected in 77,7\% of urine cultures performed after the biopsy. The most commonly isolated microorganisms were Escherichia coli (71.4\%), Klebsiella pneumoniae (20\%), and Staphylococcus spp (8.5\%), respectively. Blood culture was obtained from $37.7 \%$ of the patients, and the reproduction rate was $64.7 \%$. E. coli was the most frequently isolated agent in blood culture. In urine culture, methicillin resistance in staphylococci was 66.6\%, Extended Spectrum Beta-Lactamase (ESBL) rate in E. coli was 56\%, ciprofloxacin resistance was 91,4\%, trimethoprim- sulfamethoxazole resistance was $54.2 \%$ and gentamicin resistance was $25.7 \%$.

Discussion and Conclusion: Nearly all patients who developed urinary tract infection after prostate biopsy had ciprofloxacinresistant microorganisms in urine cultures. Targeted antibiotic use can be considered to reduce post- biopsy infection. Also, alternative agents as prophylaxis can be used.
\end{abstract}

Keywords: Ciprofloxacin; prostate biopsy; prophylaxis; urinary tract infection.

U Itrasound-guided prostate biopsy is the standard method for the diagnosis of prostate cancer ${ }^{[1]}$. However, after prostate biopsy, hematuria, urinary retention, as well as infectious complications, such as acute urinary tract infections (UTI), epididymitis, prostatitis and rarely urosepsis, may develop ${ }^{[2]}$. To prevent infectious complications, various prophylactic antibiotics are applied before prostate biopsy ${ }^{[1,3]}$. For this purpose, fluoroquinolones are the most commonly used prophylaxis worldwide before biopsy ${ }^{[1-3]}$. The main source of urological infections after the biopsy is contamination and inoculation from rectal flora. Most patients scheduled for biopsy have a history of recurrent

Correspondence (İletişim): Musab Ali Kutluhan, M.D. Saglik Bilimleri Universitesi Fatih Sultan Mehmet Egitim ve Arastirma Hastanesi, Istanbul, Turkey

Phone (Telefon): +90 5372826616 E-mail (E-posta): dr.musab151@gmail.com

Submitted Date (Başvuru Tarihi): 08.11.2019 Accepted Date (Kabul Tarihi): 19.11.2019

Copyright 2019 Haydarpaşa Numune Medical Journal

OPEN ACCESS This is an open access article under the CC BY-NC license (http://creativecommons.org/licenses/by-nc/4.0/) 
quinolone use ${ }^{[4]}$. Therefore, it is not surprising that the quinolone resistance of uropathogens is increased. Really soon, an increasing number of reports has been published on increased quinolone resistance in the urological population ${ }^{[5-7]}$. In this study, we aimed to investigate urinary tract infection rates after transrectal ultrasound-guided prostate biopsy and to determine antibiotic resistance profiles in urinary cultures after prostate biopsy.

\section{Materials and Methods}

Between 2015 and 2019, 892 patients who underwent prostate biopsy because of suspected rectal examination and/or increased PSA level were retrospectively reviewed and included in our study. Before the biopsy, urine cultures and microscopic analysis of the urine were performed from all patients. Oral ciprofloxacin was prescribed for seven days for prophylaxis (500 $\mathrm{mg}$ bid starting three days before the biopsy). Bowel cleaning enemas were used the night before the biopsy. No other cleaning agents, such as povidone-iodine, were used before the biopsy. To reduce pain rectal, lidocaine gel applied and also ultrasound- guided periprostatic nerve blockage was performed using $10 \mathrm{ml}$ bupivacaine. The procedure was performed with a left lateral decubitus position. Standard 12 quadrant prostate biopsies were performed to all patients. There were 45 patients who were applied to the hospital after prostate biopsy with complaints of dysuria, fever or sepsis findings. In these cases, microorganisms growing in urine culture and resistance status were also investigated. Patients who did not use prophylactic antibiotics and/or bowel cleaning enemas before biopsy were excluded from this study. Also, patients who did not have sterile urine culture before biopsy were not included in our study. For statistical analysis of data, SPSS 22 program was used. Data were expressed as mean \pm standard deviation or as a number (percentage).

\section{Results}

A total of 45 male patients who had urinary tract infection after biopsy were included in this study. The mean age of the patients was $59.57 \pm 9.94$ years. Table 1 shows the general demographic characteristics of the patients. Forty patients

Table 1. General demographic characteristics of the patients

\begin{tabular}{lc} 
& Patients $(\mathbf{n}=\mathbf{4 5})$ \\
\hline Age (Mean \pm SD) & $59.57 \pm 9.94$ \\
DM (\%) & 24 \\
Prostate abscess & 0 \\
\hline
\end{tabular}

DM; Diabetes Mellitus. had complaints of dysuria, 17 had a fever with tremors, and one patient had sepsis. $24 \%$ of patients had diabetes mellitus. No prostate abscess was detected on ultrasound. The overall rate of urinary tract infection after ciprofloxacin prophylaxis was $5.04 \%$ and $1.90 \%$ of the patients had fever after prostate biopsy. Reproduction was detected in 77.7 of urine cultures of patients who were applied to the hospital after prostate biopsy with complaints of dysuria, fever or sepsis findings. The most commonly isolated microorganisms were Escherichia coli (E. Coli) (71.4\%), Klebsiella pneumonia (20\%), and Staphylococcus spp (8.5\%), respectively. Blood culture was obtained from $37.7 \%$ of the patients, and the reproduction rate was $64.7 \%$. E. coli was the most frequently isolated agent in blood culture. In urine culture, methicillin resistance in staphylococci was $66.6 \%$, Extended Spectrum Beta-Lactamase (ESBL) rate in E. coli was $56 \%$, ciprofloxacin resistance was $91.4 \%$, trimethoprimsulfamethoxazole resistance was $54.2 \%$ and gentamicin resistance was $25.7 \%$. Table 2 shows urine culture results and resistance status in patients with urinary tract infection after prostate biopsy. There was ESBL positive E.coli growth in blood and urine culture of the patient who had sepsis and the patient was treated with appropriate antibiotherapy and survived.

\section{Discussion}

Transrectal ultrasound-guided prostate biopsy is a frequently applied, reliable and well-tolerated method ${ }^{[8]}$. However, unwanted complications can be seen despite the precautions taken ${ }^{[9]}$. Urinary tract infection is one of the most common complications causing significant morbidity after prostate biopsy. Antimicrobial prophylaxis to prevent this complication is recommended as a standard procedure ${ }^{[10]}$. However, the choice of regimens and duration of prophylaxis remains arguable. EAU and AUA guidelines

Table 2. Urine culture results and resistance status in patients with urinary tract infection after prostate biopsy

\begin{tabular}{lcc}
\hline & $\begin{array}{c}\text { Urine culture } \\
(\mathbf{n = 4 5 )}(\mathbf{1 0 0 \% )}\end{array}$ & $\begin{array}{c}\text { Blood culture } \\
\text { (n=17) (37.7\%) }\end{array}$ \\
\hline Reproduction rate (\%) & 77.7 & 64.7 \\
Escherichia coli (\%) & $71.4(56 \% \mathrm{ESBL})$ & $72.7(75 \% \mathrm{ESBL})$ \\
Klebsiellapneumoniae (\%) & $20(57.1 \% \mathrm{ESBL})$ & $27.2(66.6 \% \mathrm{ESBL})$ \\
Staphylococcus spp (\%) & $8.5(66.6 \% \mathrm{MRSA})$ & 0 \\
ciprofoxacillin resistance (\%) & 91.4 & 82.3 \\
Trimethoprim-sulfometaxazole & 54.2 & 58.8 \\
Gentamisin & 25.7 & 35.2 \\
\hline
\end{tabular}

ESBL; Extended spectrum beta lactamase. 
on prostate biopsies differ concerning antibiotic choices for prophylaxis ${ }^{[11,12]}$. Most commonly used agent as prophylaxis is fluoroquinolones ${ }^{[13]}$. In our study, ciprofloxacin was prescribed prophylactically to the patients before prostate biopsy as well. However, over the years due to the increased prevalence of fluoroquinolones resistance (FQ$\mathrm{R}$ ) bacteria in intestinal flora, there is a significant increase in fluoroquinolones resistance. The first study to evaluate this prevalence was performed by Batura et al. in the United Kingdom in $2010^{[14]}$. According to this study, 10,6\% of patients who had prostate biopsy had ciprofloxacin resistance coliforms in the rectal swab. In a study conducted in our country in $2014, \mathrm{FQ}-\mathrm{R}$ prevalence rate was $16 \%{ }^{[15]}$. The last study in literature which investigated FQ-R prevalence in rectal swab was conducted by Würnschimmel et al. in Germany in 2019. According to this study, FQ- R E.coli was found in $12(6 \%)$ patients out of 200 patients ${ }^{[16]}$. In our study we did not have rectal swab, so we do not have an $F Q-R$ prevalence rate in the intestinal flora.

Many different microorganisms may cause infectious complications after prostate biopsy. E. coli is the most commonly isolated bacteria in urine cultures after prostate biopsy ${ }^{[17]}$. Growing fluoroquinolone resistance has recently led to increasing rates of infective complications. In multi-center studies conducted in our country, quinoloneresistant E.coli strains in urine cultures of patients evaluated with urinary tract infection have been found between 8.3 and 38 percent ${ }^{[18,19]}$. In another study conducted in our country by Kandemir et al., ${ }^{[20]} 83.8 \%$ of the patients had fluoroquinolone resistance. Also, a significant increase in antibiotic resistance after 2008 was implicated in this study. In our study, ciprofloxacin resistance was $91.4 \%$. This rate was much higher in our study when compared to literature. This may be related to the inclusion of the patients who had a history of frequent fluoroquinolone use before prostate biopsy.

Pain and bleeding are common complications after transrectal prostate biopsy. The risk of major complications (such as sepsis), however, is very small. The incidence of infectious complications after transrectal prostate biopsy varies among studies, with a reported hospitalization rate of $0-6.3 \%{ }^{[17]}$. Choi et al. ${ }^{[21]}$ showed that the rate of febrile urinary tract infection was 3.1\% after prostate biopsy. In the same study, E.coli was the most common microorganism in urine culture. As in our study, fluoroquinolone resistance was found to be high in this study (82\%). In our study, the rate of infectious complications was 5.04\%.

Bacteria in the rectal flora are inoculated into the prostate and blood during prostate biopsy. Therefore, bacteria and their antibiotic resistance patterns in rectal flora may be important. Although it has not been confirmed that bacteria present in the rectal flora cause infection after prostate biopsy, the presence of resistant bacteria in the intestinal flora may be considered to increase the risk of infection with resistant bacteria. To reduce infection rates, targeted antibiotics have been used. In a study conducted by Doherty et al., post-biopsy infection occurred in one (2\%) patient in the targeted antibiotic group, and five (10\%) patients in the empirical antibiotic group ${ }^{[22]}$. Another study conducted by Liss et al. ${ }^{[23]}$ also showed that the rate of post-biopsy sepsis was lower in the targeted antibiotic group than the empirical antibiotic group ( $0.44 \%$ vs. $0.56 \%)$. According to the literature, targeted antibiotic use seems to have lower postbiopsy infection rates. Thus, rectal swab could be evaluated before prostate biopsy.

Our study has some limitations. Although information regarding antibiotic use was obtained verbally from patients and confirmed using the hospital records system, there remains the possibility that some drugs were taken without a prescription or the patient did not recall taking them. Also, resistance rates may also vary depending on the culture methods.

\section{Conclusion}

In conclusion, in our study, nearly all patients who developed urinary tract infection after prostate biopsy had ciprofloxacin-resistant microorganisms in urine cultures. FQ-resistance is still increasing due to the overuse of this group of antibiotics. Targeted antibiotic use can be considered to reduce post- biopsy infection. Also, alternative agents as prophylaxis can be used.

Ethical Committee Approval: Retrospective study.

Peer-review: Externally peer-reviewed.

Authorship Contributions: Concept: M.A.K., Design: T.T., Supervision: T.T., Materials: T.T., Data collection and processing: M.A.K., Analysis and/ or interpretations: M.A.K., Litarature search: M.A.K., Writing: M.A.K., Critical review: R.T.

Conflict of Interest: None declared.

Financial Disclosure: The authors declared that this study received no financial support.

\section{References}

1. Ramey J, Halpern E, Gomella J. Ultrasonography and biopsy of the prostate. Campbell-Walsh Urology. Philadelphia: Saunders; 2007.

2. Simsir A, Kismali E, Mammadov R, Gunaydin G, Cal C. Is it 
possible to predict sepsis, the most serious complication in prostate biopsy? Urol Int 2010;84:395-9. [CrossRef]

3. Zaytoun OM, Vargo EH, Rajan R, Berglund R, Gordon S, Jones JS. Emergence of fluoroquinolone-resistant Escherichia coli as cause of postprostate biopsy infection: implications for prophylaxis and treatment. Urology 2011;77:1035-41. [CrossRef]

4. Hamarat MB, Tarhan F, Horuz R, Öcal GA, Demirkol MK, Kafkaslı $A$, et al. Infective complications in patients after transrectal ultrasound-guided prostate biopsy and the role of ciprofloxacin resistant Escherichia coli colonization in rectal flora. Turk J Urol 2017;43:210-25. [CrossRef]

5. Ismail M, Saini A, Nigam R. Ciprofloxacin-resistant infection after transrectal ultrasonography-guided prostate biopsy: should we reassess our practice? BJU Int 2011;108:305-6.

6. Rudzinski JK, Kawakami J. Incidence of infectious complications following transrectal ultrasound-guided prostate biopsy in Calgary, Alberta, Canada: A retrospective population-based analysis. Can Urol Assoc J 2014;8:E301-5. [CrossRef]

7. Liss MA, Chang A, Santos R, Nakama-Peeples A, Peterson EM, Osann K, et al. Prevalence and significance of fluoroquinolone resistant Escherichia coli in patients undergoing transrectal ultrasound guided prostate needle biopsy. J Urol 2011;185:1283-8. [CrossRef]

8. Lee SH, Chen SM, Ho CR, Chang PL, Chen CL, Tsui KH. Risk factors associated with transrectal ultrasound guided prostate needle biopsy in patients with prostate cancer. Chang Gung Med J 2009;32:623-7.

9. Efesoy O, Bozlu M, Çayan S, Akbay E. Complications of transrectal ultrasound-guided 12-core prostate biopsy: a single center experience with 2049 patients. Turk J Urol 2013;39:611. [CrossRef]

10. Enlund AL, Varenhorst E. Morbidity of ultrasound-guided transrectal core biopsy of the prostate without prophylactic antibiotic therapy. A prospective study in 415 cases. Br J Urol 1997;79:777-80. [CrossRef]

11. Bonkat G, Bartoletti RR, Bruyere B, Cai T, Geerlings SE, Köves B, et al. European Urology Guidelines Urological infections: Periprocedural antibiotic prophylaxis. 2019.

12. Bertsch J, Hitt E, Hubbard H, Pope S, Wilder V. The Prevention and Treatment of the More Common Complications Related to Prostate Biopsy Update. 2016.

13. Lista F, Redondo C, Meilán E, García-Tello A, Ramón de Fata $F$, Angulo JC. Efficacy and safety of fosfomycin-trometamol in the prophylaxis for transrectal prostate biopsy. Prospective randomized comparison with ciprofloxacin. Actas Urol Esp
2014;38:391-6. [CrossRef]

14. Batura D, Rao GG, Nielsen PB. Prevalence of antimicrobial resistance in intestinal flora of patients undergoing prostatic biopsy: implications for prophylaxis and treatment of infections after biopsy. BJU Int 2010;106:1017-20. [CrossRef]

15. Tukenmez Tigen E, Tandogdu Z, Ergonul O, Altinkanat G, Gunaydin $B$, Ozgen $M$, et al. Outcomes of fecal carriage of extended-spectrum $\beta$-lactamase after transrectal ultrasoundguided biopsy of the prostate. Urology 2014;84:1008-15.

16. Würnschimmel C, Busto Martin L, Leyh-Bannurah SR, OhHohenhorst SJ, Kachanov M, Maurer T, et al. Rectal Swabs for Detecting Multidrug Resistant Bacteria Prior to Transrectal Prostate Fusion Biopsy: A Prospective Evaluation of Risk Factor Screening and Microbiologic Findings. Urology 2020;136:127-32. [CrossRef]

17. Loeb S, Vellekoop A, Ahmed HU, Catto J, Emberton M, Nam R, et al. Systematic review of complications of prostate biopsy. Eur Urol 2013;64:876-92. [CrossRef]

18. Arslan H, Azap OK, Ergönül O, Timurkaynak F; Urinary Tract Infection Study Group. Risk factors for ciprofloxacin resistance among Escherichia coli strains isolated from community-acquired urinary tract infections in Turkey. J Antimicrob Chemother 2005;56:914-8. [CrossRef]

19. Atılgan D, Gençten Y, Kölükçü E, Kılıç Ş, Uluocak N, Parlaktaş BS, et al. Comparison between ciprofloxacin and trimethoprim-sulfamethoxazole in antibiotic prophylaxis for transrectal prostate biopsy. Turk J Urol 2015;41:27-31. [CrossRef]

20. Kandemir Ö, Bozlu M, Efesoy O, Güntekin O, Tek M, Akbay E. The incidence and risk factors of resistant $E$. coli infections after prostate biopsy under fluoroquinolone prophylaxis: a single-centre experience with 2215 patients. J Chemother 2016;28:284-8. [CrossRef]

21. Choi JW, Kim TH, Chang IH, Kim KD, Moon YT, Myung SC, et al. Febrile urinary tract infection after prostate biopsy and quinolone resistance. Korean J Urol 2014;55:660-4. [CrossRef]

22. Doherty AF, Ikuerowo SO, Jeje EA, Ibrahim NA, Ojongbede OL, Mutiu WB, et al. A prospective randomized comparative study of targeted versus empirical prophylactic antibiotics in the prevention of infective complications following transrectal ultrasound-guided prostate biopsy. Ann Afr Med 2019;18:1327. [CrossRef]

23. Liss MA, Kim W, Moskowitz D, Szabo RJ. Comparative Effectiveness of Targeted vs Empirical Antibiotic Prophylaxis to Prevent Sepsis from Transrectal Prostate Biopsy: A Retrospective Analysis. J Urol 2015;194:397-402. [CrossRef] 